\title{
Dose-sliced data collection; Get the best from that crystal!
}

Kanagalaghatta Rajashankar, Igor Kourinov, Jon Schuerman, Frank Murphy and Malcolm Capel NE-CAT and Department of Chemistry and Chemical Biology, Cornell University, Argonne National Laboratory, Argonne, IL 60439, USA

Data collection is the final and important experimental step in any X-ray structure determination project. Choice of optimal values for data collection parameters results in optimum data. In a diffraction data collection experiment, the parameters that need optimization include crystal-to-detector distance, rotation per frame, total rotation range and exposure time per frame. Some of these parameters can be determined from known crystal orientation and some have to be determined empirically. Exposure time falls into the second category and together with the incident X-ray flux, it determines the total X-ray dose per frame. Too high dose will result in radiation damage, affecting data accuracy and completeness. At the same time, too little dose will negatively affect the diffraction strength and data resolution, hence will not make use of the full diffraction potential of the crystal. Therefore, one has to optimize the exposure time so that completeness, redundancy and resolution are all maximized simultaneously.

We propose that optimum exposure time can be achieved by a method we termed "doseslicing”. This method involves collecting $\mathrm{N}$ sweeps of data, each with a very low-dose. For $\mathrm{N}$ measurements, the signal-to-ratio is $\sqrt{ } \mathrm{N}^{*}<\mathrm{I}>/ \sigma$, where $<\mathrm{I}>$ is the average intensity for a single sweep. As $\mathrm{N}$ increases, the signal-to-noise ratio improves continuously. At the same time, the crystal is suffering radiation damage during the course of data collection, which will be degrading the signal-to-noise. Hence, after a certain number of $\mathrm{M}$ sweeps, the gain in signal-tonoise (by increased $\mathrm{N}$ ) is cancelled out by radiation damage, resulting in no improvement in data quality. Eventually, the data degradation due to radiation damage will be so severe that it surpasses the gains in signal-to-noise with increasing N. Hence, the best data would come be from the first $\mathrm{M}$ complete rotations, discarding the data from last N-M rotations. Pixel Array Detectors are better suited for these experiments than CCD detectors, because of their fast and noiseless readout. Details of some real data sets collected using this method will be discussed during the meeting.

Acta Cryst. (2018). A74, a309 